\title{
Origen anómalo de la arteria coronaria derecha de la arteria pulmonar en un lactante con soplo cardíaco. A propósito de un caso
} Anomalous origin of the right coronary artery from the pulmonary artery in an infant with a heart murmur. Case report

\author{
Dra. Melina J. Saavedra ${ }^{a}$ Dr. Javier Mozzi ${ }^{a}$, Dra. Natalia Nápoli ${ }^{a}$, Dra. Alejandra Villa ${ }^{a}$ Dr. Jorge Barretta ${ }^{b}$ \\ Dr. Pablo Marantz ${ }^{a}$
}

\begin{abstract}
RESUMEN
El origen anómalo de la arteria coronaria derecha de la arteria pulmonar es una entidad rara con una incidencia del $0,002 \%$ y potencialmente grave, que puede causar muerte súbita. El diagnóstico frecuentemente es incidental, secundario a evaluaciones por soplos cardíacos a diferencia del origen anómalo de la arteria coronaria izquierda de la arteria pulmonar, reconocido por presentar isquemia miocárdica e insuficiencia cardíaca.

Se reporta el caso de una niña de 6 meses evaluada por un soplo y derivada por sospecha de fístula coronaria.

La evaluación mediante ecocardiograma doppler color, cateterismo cardíaco y angiotomografía mostró la presencia de una comunicación interauricular ostium secundum pequeña y origen anómalo de la arteria coronaria derecha de la arteria pulmonar. A los 11 meses, se realizó una cirugía de reimplante de la arteria coronaria derecha en la arteria aorta y el cierre de la comunicación interauricular.

Palabras clave: ARCAPA, anomalías de los vasos coronarios, ALCAPA, isquemia miocárdica, muerte súbita.
\end{abstract}

http: / / dx.doi.org/10.5546/ aap.2018.e789

Texto completo en inglés:

http: / / dx.doi.org/10.5546/ aap.2018.eng.e789

Cómo citar: Saavedra MJ, Mozzi J, Nápoli N, Villa A, et al. Origen anómalo de la arteria coronaria derecha de la arteria pulmonar en un lactante con soplo cardíaco. A propósito de un caso. Arch Argent Pediatr 2018;116(6):e789-e792.

a. Departamento de Pediatría, Servicio de Cardiología Pediátrica.

b. Servicio de Cirugía Cardiovascular Pediátrica.

Hospital Italiano de Buenos Aires.

Correspondencia:

Dra. Melina J. Saavedra: melina.saavedra@hiba.org.ar

Financiamiento: Ninguno.

Conflicto de intereses: Ninguno que declarar.

Recibido: 17-5-2018

Aceptado: 31-7-2018

\section{INTRODUCCIÓN}

Las anomalías del origen de las arterias coronarias son infrecuentes. ${ }^{1}$ El origen anómalo de la arteria coronaria derecha de la arteria pulmonar (anomalous origin of the right coronary artery from the pulmonary artery; ARCAPA, por sus siglas en inglés) es una entidad rara, con una incidencia del $0,002 \% .{ }^{2}$ Un tercio de los pacientes presenta otras anomalías cardíacas asociadas; las más frecuentes son la ventana aortopulmonar y la tetralogía de Fallot..$^{2-5}$

Se describieron, en la literatura, al menos, 100 casos de ARCAPA. El $50 \%$ fueron diagnosticados de forma incidental en el contexto de una evaluación por soplo cardíaco y se observaron, en algunos pacientes, signos de isquemia en el electrocardiograma. ${ }^{2-4,6,7}$

Las anomalías de implantación coronaria son entidades potencialmente graves que pueden ser causa de muerte súbita, por lo que la corrección quirúrgica estaría indicada aun en pacientes asintomáticos. ${ }^{4,7-9}$

\section{REPORTE DE CASO}

Se reporta el caso de una niña de 6 meses de edad asintomática, evaluada por un soplo en la ciudad de origen y derivada por la sospecha de fístula coronaria.

Previamente sana, a los 4 meses, estuvo internada por bronquiolitis con hipoxemia. En el examen cardiovascular, presentaba un soplo sistólico eyectivo en el mesocardio de intensidad $2 / 6$, por lo que se solicitó un ecocardiograma y se diagnosticó comunicación interauricular ostium secundum (CIAOS) y sospecha de fístula coronaria. Presentaba peso en el percentil 97 y talla en el percentil 6,7 según las tablas de la Organización Mundial de la Salud.

En nuestro Servicio, se realizó un ecocardiograma doppler color, y se constataron ambas coronarias dilatadas, imagen sugestiva de múltiples fístulas al ventrículo derecho y una 
pequeña CIAOS sin dilatación de las cavidades derechas.

Se realizó un cateterismo cardíaco en el que no se observó la emergencia de la coronaria derecha en el origen aórtico durante la inyección de la sustancia de contraste (Figura 1). Se constató la presión diastólica levemente elevada en la arteria pulmonar $(25 / 16 \mathrm{mmHg}$, media de $19 \mathrm{mmHg})$

\section{Figura 1. Cateterismo cardíaco}

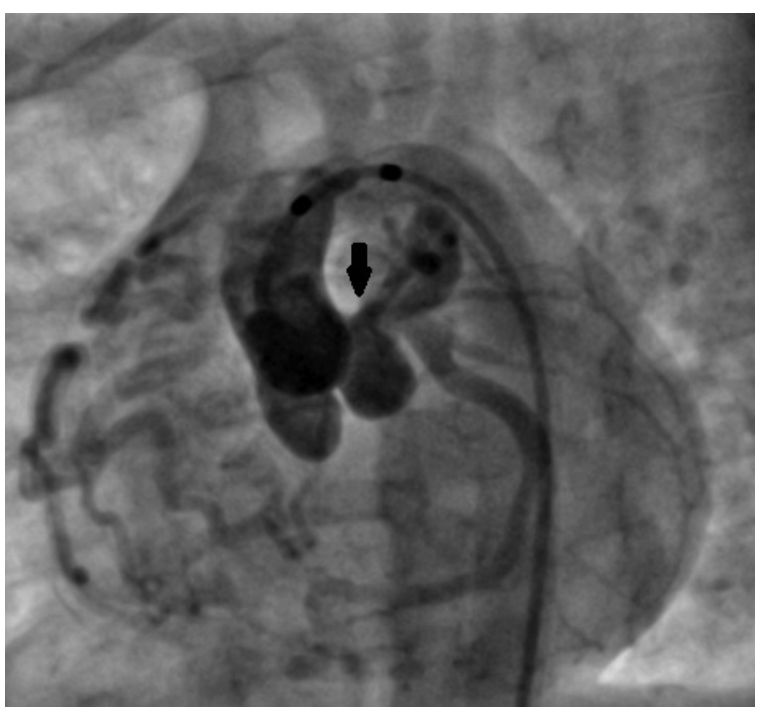

Coronaria izquierda dilatada (flecha negra). No se observa la coronaria derecha emergiendo de su seno correspondiente. Desarrollo de circulación colateral con presión de fin de diástole del ventrículo izquierdo de $12 \mathrm{mmHg}$. La arteria coronaria izquierda se encontraba dilatada con conexión al territorio de la coronaria derecha y posterior drenaje en el tronco de la arteria pulmonar. Presentaba un leve aumento del flujo pulmonar con Qp/Qs 1,2:1. Durante la inyección de la sustancia de contraste en la arteria pulmonar, no se logró apreciar con certeza el origen de la arteria coronaria derecha.

Dada la complejidad diagnóstica, se indicó, para completar la delineación de la anatomía coronaria, una angiotomografía multislice. Se evidenció el nacimiento anómalo de la coronaria derecha en la arteria pulmonar, a 8,6 $\mathrm{mm}$ del plano valvular, en la cara anterior y derecha. Esta transcurría por delante de la aorta y se dirigía al surco auriculoventricular derecho. La arteria coronaria izquierda era dominante y nacía normalmente del seno aórtico coronariano izquierdo. Se observó abundante desarrollo de circulación colateral entre las arterias descendente anterior y circunfleja con la arteria coronaria derecha. De este modo, se confirmó el diagnóstico de ARCAPA (Figura 2).

Con los resultados de los estudios complementarios, se presentó en un ateneo interdisciplinario clínico-quirúrgico y se discutieron las estrategias terapéuticas. Se decidió optar por la corrección quirúrgica.

El electrocardiograma previo a la cirugía, se encontraba dentro de los límites normales.

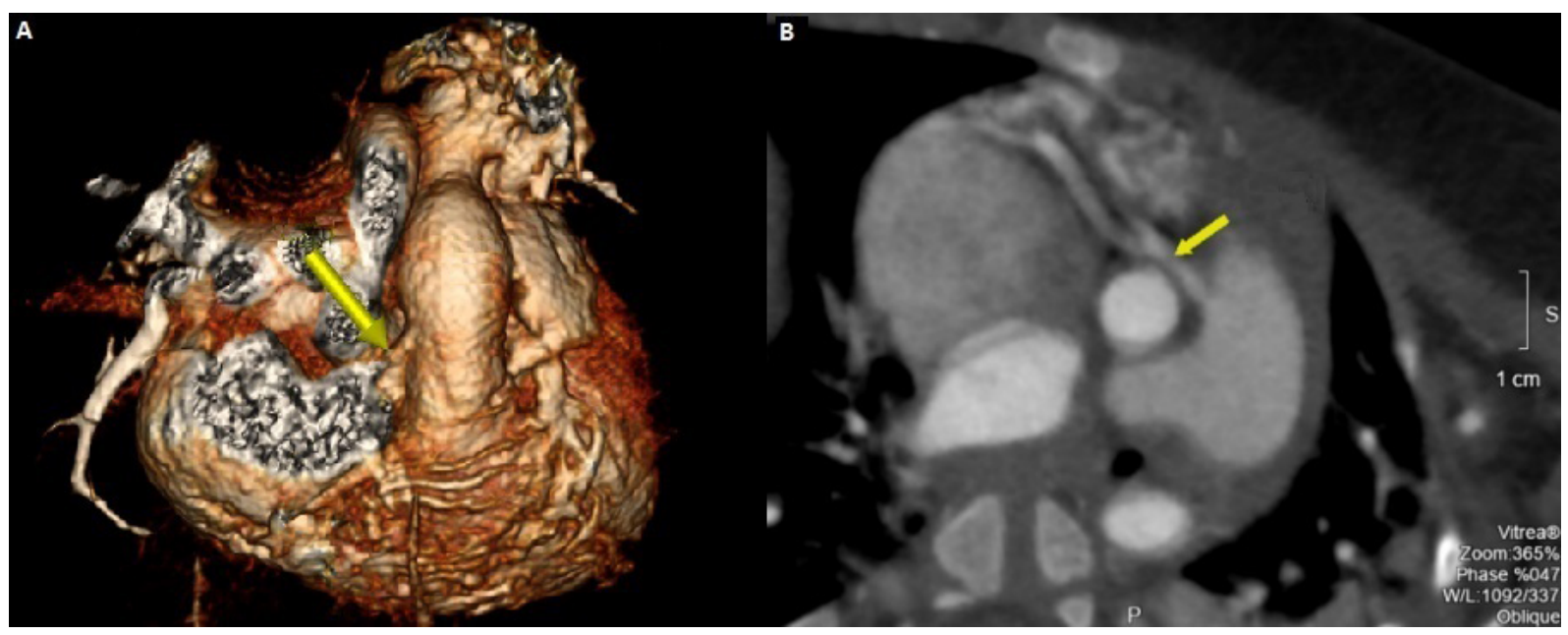

A. Reconstrucción 3D en la que se observa la arteria coronaria derecha emergiendo de la arteria pulmonar

B. Eje corto de los grandes vasos. Arteria coronaria derecha que nace de la arteria pulmonar 
A los 11 meses de edad, se realizó la cirugía de reimplante de la arteria coronaria derecha en la arteria aorta y el cierre de la CIA. Se realizó un ecocardiograma transesofágico intraoperatorio, que informó coronaria izquierda dilatada que nacía de la aorta y coronaria derecha, del tronco de la arteria pulmonar en posición lateral y a la derecha; se observaba abundante circulación colateral (Figura 3). La cirugía tuvo una duración de 207 minutos. Requirió una bomba de circulación extracorpórea por 66 minutos y clampeo aórtico durante 35 minutos. Se utilizó como soporte inotrópico milrinona $(0,5 \mathrm{mcg} / \mathrm{kg} /$ min) y adrenalina $(0,05 \mathrm{mcg} / \mathrm{kg} / \mathrm{min})$. Se logró el destete del respirador y del goteo de adrenalina a las 5 horas del posoperatorio. Permaneció con milrinona por $48 \mathrm{~h}$, y se inició luego enalapril.

La paciente no presentó complicaciones posoperatorias. El ecocardiograma mostró arteria coronaria derecha implantada en la arteria aorta con flujo laminar con función biventricular conservada. $\mathrm{Al}$ tercer día posoperatorio, se otorgó el egreso institucional medicada con enalapril $(0,1 \mathrm{mg} / \mathrm{kg} /$ día $)$ y furosemida $(2 \mathrm{mg} / \mathrm{kg} /$ día), y continuó, posteriormente, con controles ambulatorios.

\section{DISCUSIÓN}

Las anomalías congénitas de las arterias coronarias son un conjunto de variantes anatómicas vasculares presentes desde el nacimiento. El ARCAPA es una anomalía coronaria infrecuente, descrita, por primera vez, en el año 1885 por Brooks. ${ }^{10}$ Puede estar presente en corazones estructuralmente normales $\mathrm{o}$, en un $30 \%$, asociado a otras cardiopatías congénitas; las más comunes son la ventana aortopulmonar y la tetralogía de Fallot. ${ }^{2-5}$ En nuestro caso, la paciente presentaba ARCAPA asociado a CIA, que ha sido reportada con menor frecuencia.
Al día de hoy, se describieron, en la literatura, al menos, 100 casos de ARCAPA, que incluyen a pacientes pediátricos y adultos con amplia variedad de manifestaciones clínicas y métodos diagnósticos. ${ }^{5}$ En los pacientes publicados, la mayoría fueron diagnosticados de forma incidental en el contexto de una evaluación por un soplo cardíaco. ${ }^{2,4,6,7}$ A diferencia del origen anómalo de la arteria coronaria izquierda de la arteria pulmonar (anomalous origin of the left coronary artery from the pulmonary artery; ALCAPA, por sus siglas en inglés), conocido por las manifestaciones clínicas a temprana edad con insuficiencia mitral y signos de infarto de miocardio anterolateral, la mayoría de los pacientes con ARCAPA no habían desarrollado síntomas. Sin embargo, algunos presentaban signos de isquemia miocárdica en el electrocardiograma, al momento del diagnóstico. ${ }^{3}$

En nuestro caso, el diagnóstico se realizó en una paciente asintomática, que fue evaluada por la presencia de un soplo cardíaco en la auscultación y que no presentaba signos de isquemia en el electrocardiograma.

Menos frecuentemente, el ARCAPA puede desarrollarse de forma sintomática, debido al fenómeno de robo coronario que se produce desde la coronaria izquierda hacia la derecha a través de vasos colaterales. En este caso, los pacientes pueden presentar síntomas de isquemia miocárdica, como precordialgia, disnea, fatiga, insuficiencia cardíaca congestiva, infarto de miocardio, incluso muerte súbita. ${ }^{4-6,8}$ La gravedad depende del impacto de la anomalía en la entrega de oxígeno al miocardio, dado por el tipo de anomalía, la dirección del flujo y la formación de circulación colateral. ${ }^{2,4,6,7}$

Existen situaciones protectoras asociadas al ARCAPA, como la estenosis del origen de la arteria coronaria derecha anómala, la

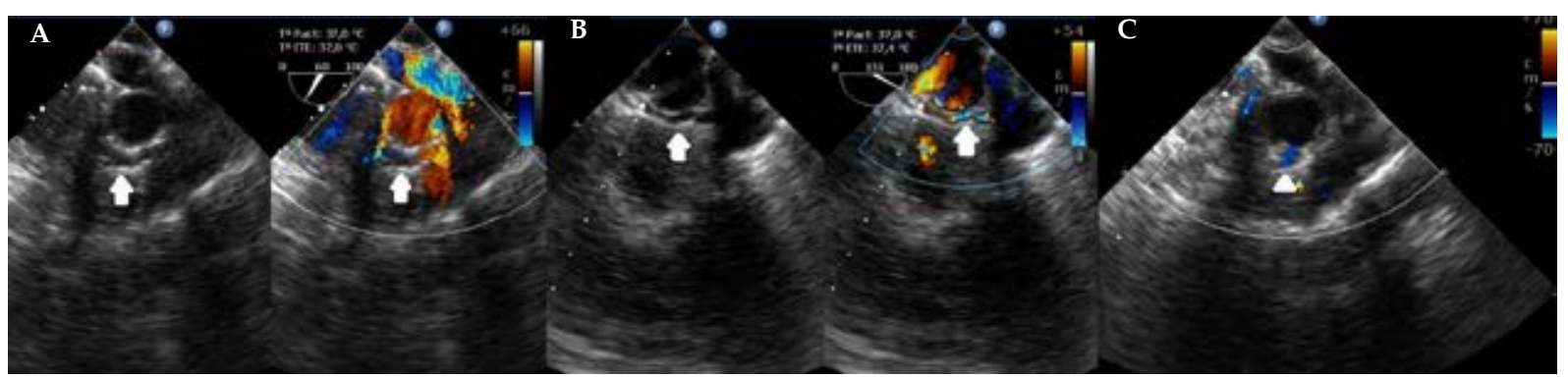

A y B. Se observa la arteria coronaria derecha emergiendo de la arteria pulmonar (flecha blanca)

C. Posoperatorio inmediato con reimplante de la arteria coronaria derecha en la arteria aorta (punta de flecha blanca) 
dominancia coronaria izquierda y la presencia de cortocircuitos de izquierda a derecha (defectos interventriculares, ductus arterioso persistente o ventana aortopulmonar). ${ }^{2,11} \mathrm{El}$ aumento de la presión en la arteria pulmonar se asocia con un menor robo de la circulación coronaria hacia la arteria pulmonar y, a la vez, esta condición puede brindar mayor flujo anterógrado desde la arteria pulmonar hacia la arteria coronaria derecha. ${ }^{11}$ En nuestra paciente, la dominancia coronaria izquierda podría haber sido protectora.

Los métodos diagnósticos dependen del período histórico de los casos reportados. Antes de 1985, mayormente, el diagnóstico se realizaba mediante autopsia, angiografía o durante la cirugía cardiovascular. ${ }^{2,4}$ En la actualidad, con el ecocardiograma doppler color, se puede arribar al diagnóstico del ARCAPA. Los hallazgos característicos son la dilatación de la coronaria izquierda, el flujo a través de la circulación colateral con incorrecta apariencia de fístulas y la visualización del origen anómalo de la coronaria derecha con flujo retrógrado desde esta hasta la arteria pulmonar. ${ }^{5,6}$ Sin embargo, los métodos con representación en dos dimensiones pueden ser limitados para determinar el origen y el curso de la arteria coronaria. ${ }^{5}$ En los últimos años, con los avances en la definición de los estudios por imágenes, se puede optar por métodos no invasivos de alta precisión con representación 3D, como la angiotomografía computada y la resonancia magnética cardíaca. ${ }^{5}$

En los pacientes con diagnóstico de ARCAPA, dado el fenómeno de robo coronario con potencial isquemia miocárdica, la resolución quirúrgica con el reimplante de la arteria coronaria derecha en la arteria aorta es fuertemente recomendada, incluso en los pacientes asintomáticos. ${ }^{2,4,7,8}$ Esta técnica quirúrgica restablece el doble sistema de circulación coronaria y disminuye el riesgo de muerte súbita. ${ }^{8}$ La cirugía de reimplante de la arteria coronaria derecha y el cierre de la CIA se realizó en nuestro paciente a los 11 meses de edad sin complicaciones.

\section{CONCLUSIÓN}

El ARCAPA es una anomalía coronaria infrecuente potencialmente mortal. Dada la proporción de pacientes asintomáticos, es probable que la incidencia esté subestimada. $\mathrm{Su}$ aumento cuando existe cardiopatía congénita resalta la importancia de la adecuada identificación de las arterias coronarias en este grupo de pacientes.

En la actualidad, se puede arribar al diagnóstico con métodos no invasivos de alta precisión, como la angiotomografía o la angiorresonancia.

La corrección quirúrgica que reestablece el doble sistema de circulación coronaria está fuertemente recomendada, aun en los pacientes asintomáticos, con el fin de disminuir el riesgo de muerte súbita.

\section{REFERENCIAS}

1. Angelini P, Velasco JA, Flamm S. Coronary anomalies: incidence, pathophysiology, and clinical relevance. Circulation. 2002; 105(20):2449-54.

2. Williams IA, Gersony WM, Hellenbrand WE. Anomalous right coronary artery arising from the pulmonary artery: A report of 7 cases and a review of the literature. Am Heart J. 2006; 152(5):1004.e9-17.

3. Radke PW, Messmer BJ, Haager PK, Klues HG. Anomalous origin of the right coronary artery: preoperative and postoperative hemodynamics. Ann Thorac Surg. 1998; 66(4):1444-9.

4. Kim K, Jo E, Yu J, Kil H. Anomalous right coronary artery from pulmonary artery discovered incidentally in an asymptomatic young infant. Korean J Pediatr. 2016;59(Suppl 1):S80-3.

5. Gilmour J, Kafka H, Ropchan G, Johri AM. Anomalous right coronary artery: a multimodality hunt for the origin. Case Rep Cardiol. 2011; 2011:286598.

6. Afolabi-Brown O, Witzke C, Moldovan R, Pressman G. A Different Kind of Christmas Tree: Anomalous Origin of the Right Coronary Artery from the Pulmonary Artery (ARCAPA). Echocardiography. 2014; 31(2):e52-4.

7. García Manzano PR, Domínguez P, Merino L, et al. Origen anómalo de arteria coronaria derecha desde arteria pulmonar: reimplante directo en aorta en un niño de 8 meses de edad. Rev Fed Arg Cardiol. 2010; 39(2):133-5.

8. Bregman D, Brennan JF, Singer A, et al. Anomalous origin of the right coronary artery from the pulmonary artery. $J$ Thorac Cardiovasc Surg. 1976; 72(4):626-30.

9. Veselka J, Widimský P, Kautzner J. Reimplantation of anomalous right coronary artery arising from the pulmonary trunk leading to normal coronary flow reserve late after surgery. Ann Thorac Surg. 2003; 76(4):1287-9.

10. Brooks HS. Two cases of an abnormal coronary artery of the heart arising from the pulmonary artery: with some remarks upon the effect of this anomaly in producing cirsoid dilatation of the vessels. J Anat Physiol. 1885; 20(Pt 1):26-9.

11. Ramani J, Ananthanarayanan C, Pujara J, et al. Intramural Anomalous Right Coronary Artery From the Main Pulmonary Artery. World J Pediatr Congenit Heart Surg. 2017; 8(6):745-9. 